\title{
Neumocistosis extrapulmonar: comunicación de un caso
}

\author{
Carlos Valdebenito, Macarena Bonacic, Jennifer Matamala y Marcelo Wolff
}

\footnotetext{
Hospital San Borja Arriarán. Servicio de Infectología (MB, MW) Servicio Anatomía Patológica (JM) Universidad de Chile. Facultad de Medicina. Programa de formación en Medicina Interna (CV)

Los autores declaran no tener conflictos de interés ni fuente de financiamiento.

Recibido: 5 de agosto de 2015 Aceptado: 15 de abril de 2015

Correspondencia a: Carlos Valdebenito c.valde@gmail.com
}

\section{Extrapulmonary pneumocystosis: a case report}

We report a case of a middle-age male patient, with newly HIV infection in AIDS stage diagnosis, no comorbitidies, who was hospitalized for subacute malaise, fever, self-limited unproductive cough and no bloody chronic diarrea. The diagnosis of Pneumocystis jiroveci pneumonia was performed by imagenological suspicion and stains of cysts of this pathogen with bronchoalveolar lavage samples. Treatment was initiated with oral cotrimoxazole and starting HAART with good clinical outcome. Concomitantly, an etiologic study was conducted for chronic diarrhea and through histopathological examination of colonic mucosa, numerous extracellular cystic structures Pneumocystis characteristics were observed, performing the diagnosis of extrapulmonary pneumocystosis.

Extrapulmonary pneumocystosis is a rare cause of $P$. jiroveci infection, requires a high index of suspicion and should be approached in HIV patients with severe AIDS which is common in co-infection of various infections and is peremptory to make an etiologic diagnosis and early treatment.

Key words: Extrapulmonary pneumocystosis, Pneumocystis jiroveci, diarrhea, HIV.

Palabras clave: Neumocistosis extrapulmonar, Pneumocystis jiroveci, diarrea, VIH.

\section{Introducción}

L a neumocistosis extrapulmonar es una infección muy infrecuente, generalmente asociada a pacientes con infección por VIH en etapa de inmunocompromiso grave (SIDA) que están cursando con una neumonía por Pneumocystis jiroveci ${ }^{1}$.

La neumonía por $P$. jiroveci es una infección oportunista potencialmente mortal que puede afectar a individuos inmunocomprometidos, incluyendo pacientes con infección por $\mathrm{VIH}^{2}$ con recuento de linfocitos CD4 $<200$ céls $/ \mathrm{mm}^{3}$ e inmunocomprometidos no VIH, como pacientes con neoplasias, tratamiento con quimioterapia, hemopatías malignas, afecciones inflamatorias crónicas en tratamiento con fármacos inmunosupresores incluyendo glucocorticoides y pacientes receptores de trasplante de cualquier tipo ${ }^{3}$.

La introducción de profilaxis primaria y secundaria para la neumonía por $P$. jiroveci asociada a la terapia anti-retroviral (TARV) de alta efectividad ha significado una franca disminución en la incidencia de muchas infecciones oportunistas en personas con infección por VIH y la neumonía por $P$. jiroveci no es la excepción ${ }^{4,5}$. Sin embargo, sigue siendo una causa importante de morbilidad y mortalidad en pacientes que no reciben o no responden a TARV o que desconocen su estado de infección por VIH. En este grupo de pacientes con inmunocompromiso grave se han descrito casos de neumocistosis extrapulmonar. Puede comprometer varios órganos o tejidos, de forma contigua o no y con diseminación hematógena o linfática, siendo los ganglios linfáticos el principal sitio extrapulmonar afectado ${ }^{1,6}$.

A continuación, se presenta el caso de un paciente con infección por VIH en etapa SIDA, que consultó por fiebre y diarrea crónica, en el que se confirmó el diagnóstico de neumocistosis extrapulmonar.

\section{Caso clínico}

Varón de 41 años, con antecedentes de tabaquismo crónico, 15 paquetes-año suspendido tres meses previo a su ingreso, sin patologías crónicas conocidas. Consultó por cuadro clínico de tres meses de evolución, caracterizado por tos con expectoración mucosa escasa, asociado a sensación febril, compromiso del estado general, disminución de capacidad funcional, astenia y adinamia. Además el paciente refería deposiciones líquidas sin elementos patológicos, 4 a 5 episodios diarios, sin vómitos ni dolor abdominal. Dos semanas previas a su ingreso la diarrea se hizo más frecuente (10 episodios al día), de similares características, sin aumento de los síntomas respiratorios.

Se le realizaron exámenes tres semanas previo a su ingreso en los que destacaba un hematocrito de $35 \%$, VHS $101 \mathrm{~mm} / \mathrm{h}$, reacción de polimerasa en cadena (RPC) en deposiciones para Clostridium difficile negativo y coprocultivo negativo. Serología para VIH (ELISA) reactivo, con posterior confirmación en Instituto de Salud Pública (ISP) y recuento de linfocitos CD4 de 35 céls/ $\mathrm{mm}^{3}$; HLA-B 5701 negativo. En la tomografía axial com- 
putada (TAC) de tórax y abdomen (Figura 1) destacaba zonas en vidrio esmerilado en ambos campos pulmonares asociado a adenopatías supra e infra-diafragmáticas, menores de $2 \mathrm{~cm}$ y múltiples lesiones nodulares hepáticas mal definidas.

Consultó en el Policlínico de la Fundación Arriarán con el cuadro clínico descrito asociado a baja de peso de $15 \mathrm{~kg}$, compromiso del estado general, fiebre hasta $39^{\circ} \mathrm{C}$ y deshidratación grave al examen físico. De los exámenes realizados destacó una creatininemia de 2,0 $\mathrm{mg} / \mathrm{dL}$ y nitrógeno ureico de $38 \mathrm{mg} / \mathrm{dL}$. Fue internado con los diagnósticos de diarrea crónica con baja de peso significativa asociada a inmunosupresión severa, deshidratación e insuficiencia renal aguda. Se inició tratamiento con hidratación parenteral, cotrimoxazol $(20 \mathrm{mg} / \mathrm{kg} / \mathrm{día}$ en base a trimetroprim), azitromicina $1 \mathrm{~g}$ semanal y TARV con abacavir, lamivudina y atazanavir/ritonavir.

El paciente evolucionó satisfactoriamente, sin síntomas respiratorios, pero mantuvo entre 8 y 10 episodios de deposiciones líquidas al día, enflaquecido, sin otros hallazgos significativos al examen físico. Entre los exámenes realizados al día siguiente del ingreso presentaba un hematocrito $27,6 \%$, leucocitos 1.940 céls $/ \mathrm{mm}^{3}$, con $27,3 \%$ de linfocitos, plaquetas 191.000 céls $/ \mathrm{mm}^{3}$, VHS $>100 \mathrm{~mm} / \mathrm{h}$, PCR $0,65 \mathrm{mg} / \mathrm{dL}$, creatininemia $0,97 \mathrm{mg} /$ $\mathrm{dL}$, nitrógeno ureico $10 \mathrm{mg} / \mathrm{dL}$, albuminemia $2,6 \mathrm{mg} / \mathrm{dL}$, lactato deshidrogenasa (LDH) $200 \mathrm{mg} / \mathrm{dL}$, transaminasas normales, electrolitos plasmáticos normales. La serología para VHB y VHC fueron negativas. El examen de orina, urocultivo y hemocultivos también resultaron negativos. La radiografía de tórax presentaba un extenso infiltrado retículo-intersticial bilateral difuso, con opacidades perihiliares, sin imágenes de consolidación ni ocupación pleural. Por sospecha imagenológica de una neumonía por $P$. jiroveci se realizó una fibrobroncoscopía con lavado broncoalveolar (LBA). La vía aérea se observó de aspecto normal, sin secreciones. Los cultivos bacterianos y de Koch y las baciloscopias del LBA fueron negativos. La citología con tinción Grocott mostró un pequeño grupo de quistes compatibles con $P$. jiroveci. Se mantuvo tratamiento con cotrimoxazol iniciado al ingreso.

En forma concomitante, dentro del estudio etiológico de diarrea crónica en paciente inmunocomprometido, se realizó coprocultivo y toxina para Clostridium difficile negativos, examen parasitológico seriado de deposiciones (EPSD) con tinción de Burrows modificado sin elementos parasitarios, tinción Ziehl Neelsen modificado negativo para Isospora belli, Cyclospora spp. y Cryptosporidium spp. e inmunofluorescencia directa de deposiciones negativa para Microsporidium spp. Se realizó colonoscopía completa que demostró una úlcera cecal de $30 \mathrm{~mm}$ de diámetro mayor y pólipo sésil de $3 \mathrm{~mm}$ en colon sigmoides. El estudio histológico inicial concluyó una colitis crónica con presencia de úlceras en granulación, asociados

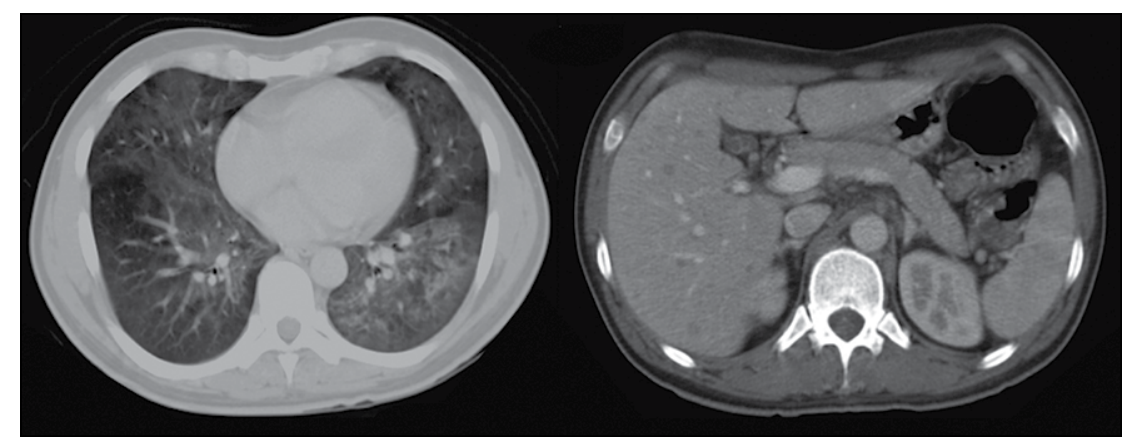

Figura 1. Tomografía axial computada (TAC) de tórax y abdomen con contraste. Áreas de vidrio esmerilado en ambos campos pulmonares con predominio en lóbulo medio e inferior izquierdo, asociado a adenopatías supra e infradiafragmáticas menores de $2 \mathrm{~cm}$. Hígado de tamaño y densidad normal con múltiples lesiones nodulares menores de $10 \mathrm{~mm}$, mal definidas.

a escasos signos de daño citopático viral por CMV y un pseudopólipo inflamatorio colónico, con resultado pendiente para tinción Ziehl Neelsen y Grocott. Además se realizó RPC para citomegalovirus (CMV) en sangre con 137 copias/mL y hemocultivos para micobacterias atípicas que fue negativo.

Debido a los hallazgos histopatológicos y el cuadro clínico inicial, se sospechó una colitis por CMV por lo que se agregó al tratamiento ganciclovir $5 \mathrm{mg} / \mathrm{kg}$, sin embargo, fue suspendido precozmente al conocer el diagnóstico histopatológico definitivo de la biopsia de colon, que informó presencia de abundantes microorganismos de tipo Pneumocystis en áreas con reacción histiocitaria prominente (Figura 2 y 3 ).

El paciente mejoró su condición clínica con franca disminución de las deposiciones líquidas al tercer día y con deposiciones normales desde el séptimo día completando 21 días de terapia con cotrimoxazol. Un nuevo TAC de abdomen y pelvis demostró una hepatomegalia asociada a múltiples lesiones hepáticas focales hipodensas de aspecto inflamatorio, menores de

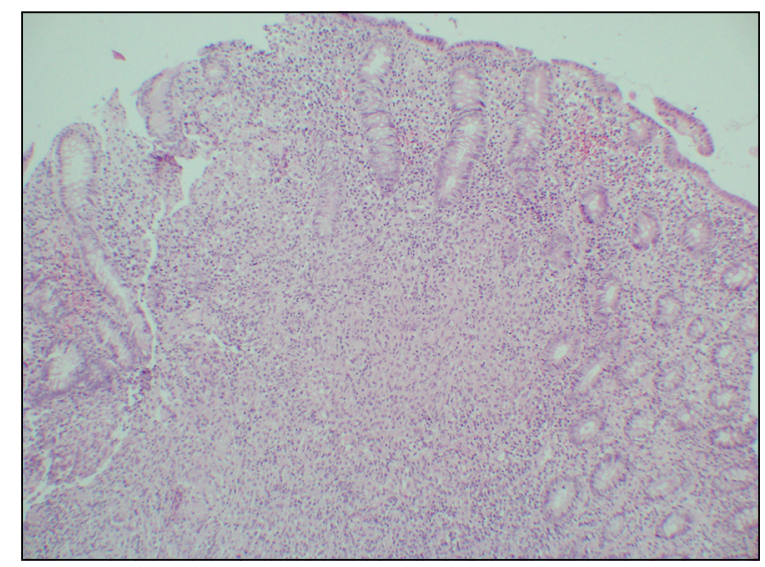

Figura 2. HE 100x, mucosa de colon sigmoides. Lámina propia con infiltrado inflamatorio crónico granulomatoso, presencia de granulomas epitelioideos esbozados no necrosantes y abundantes histiocitos. 


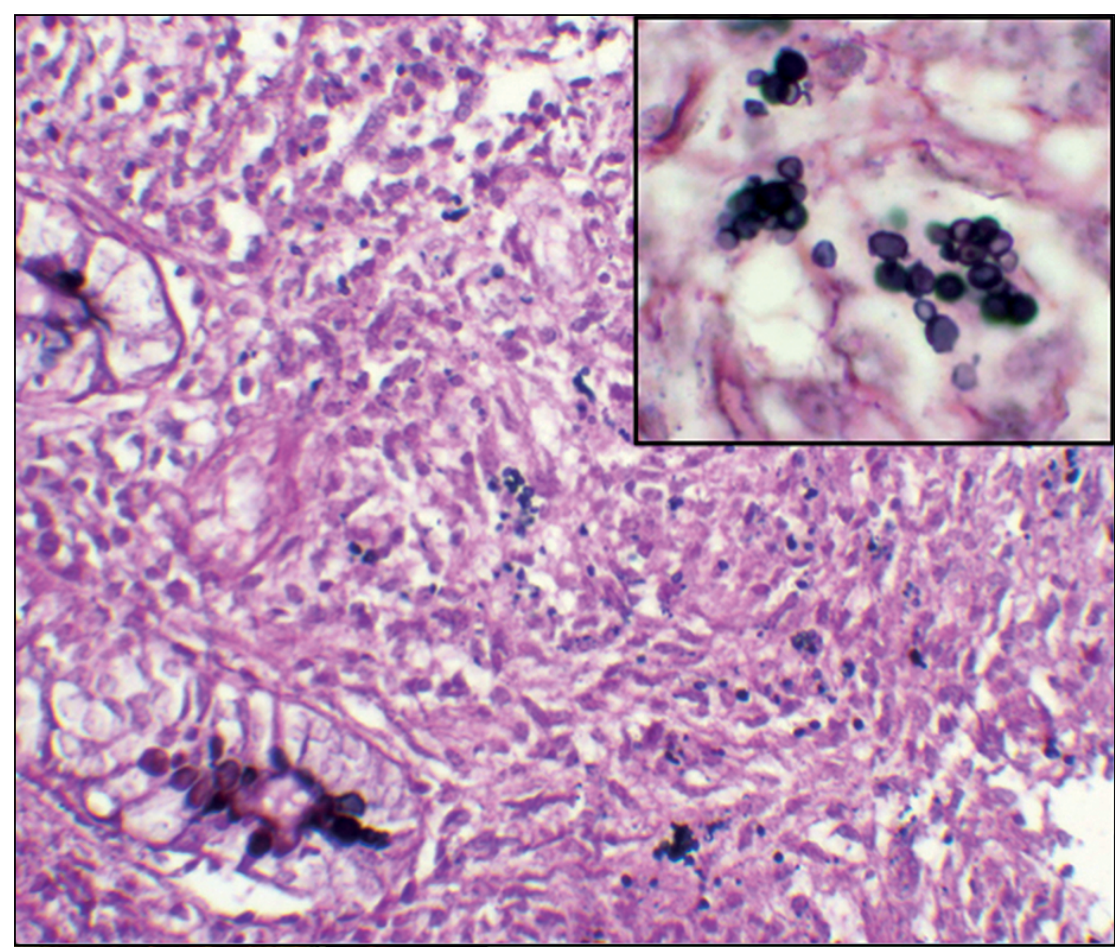

Figura 3. Grocott 400x - 1000x, mucosa de colon sigmoides. Lámina propia colónica con infiltrado inflamatorio crónico granulomatoso y numerosas estructuras quísticas extracelulares de 4 a 6 micras algunos con forma de copa.

Figura 4. TAC de abdomen y pelvis con contraste. Hígado aumentado de tamaño, de contornos regulares asociado a múltiples lesiones hepáticas focales hipodensas, mal definidas, menores de $10 \mathrm{~mm}$, de aspecto inflamatorio, posiblemente en el contexto de hepatitis granulomatosa junto a poliadenopatías de densidad homogénea en el hilio hepático, mesentéricas y retroperitoneales menores de $2 \mathrm{~cm}$.

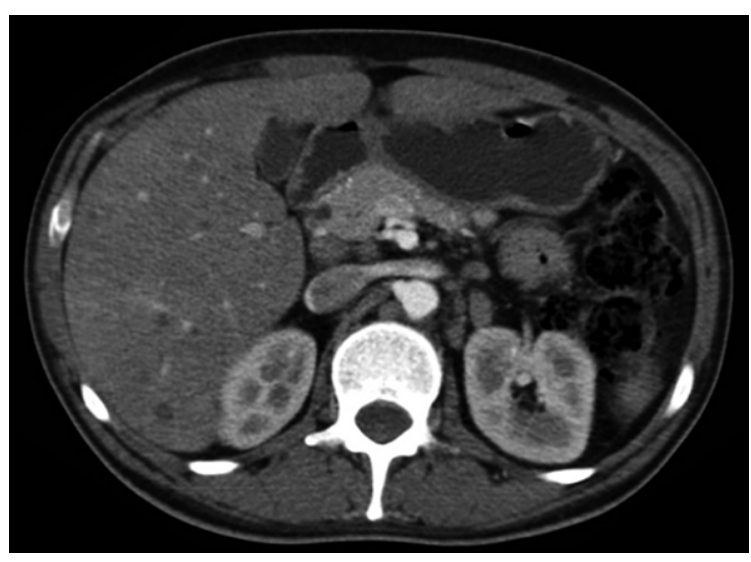

$10 \mathrm{~mm}$, posiblemente en el contexto de una hepatitis granulomatosa junto a poliadenopatías mesentéricas y retroperitoneales (Figura 4).

Finalmente se realizó el diagnóstico de una neumonía por $P$. jiroveci y una neumocistosis extrapulmonar con compromiso de colon. El paciente fue dado de alta después de un mes sin diarrea, afebril y en buenas condiciones generales. Se mantuvo TARV y profilaxis secundaria anti-Pneumocystis con cotrimoxazol. Posteriormente en el control ambulatorio posterior se objetivó un aumento de peso, ausencia de fiebre y diarrea, y carga viral en sangre indetectable.

\section{Discusión}

Pneumocystis jiroveci (ex carinii) es un organismo unicelular, ubicuo, no cultivable, extracelular, clasificado como un hongo, pero que comparte características biológicas con los protozoos ${ }^{4,7}$. Pneumocystis jiroveci continúa siendo uno de los patógenos oportunistas más importantes que afectan individuos con inmunocompromiso grave asociado a la infección por VIH u otras causas, causando una significativa morbilidad y mortalidad. Típicamente causa un cuadro de neumonía intersticial con insuficiencia respiratoria que puede ser grave y requerir apoyo ventilatorio invasor ${ }^{8}$. La presentación clínica de la neumonía por $P$. jiroveci difiere en sus características entre individuos con infección por VIH de aquellos inmunocomprometidos por otras causas ${ }^{3}$. En pacientes sin infección por VIH el diagnóstico es más difícil y con una mortalidad cercana a $40 \%$, muy superior al $10 \%$ descrito en pacientes con infección por $\mathrm{VIH}^{9}$.

El cuadro clínico más común de la neumonía por $P$. jiroveci en pacientes con infección por VIH es el inicio subagudo de disnea, fiebre, tos y malestar general, que empeora con los días o semanas. La tos es típicamente seca y no productiva. La disnea al inicio es a moderado esfuerzo, por lo que puede pasar inadvertida y progresar de modo gradual. La fatiga es una de las principales molestias, mientras que el dolor torácico, calofríos y sudoración nocturna son menos frecuentes ${ }^{2,10,11}$. En casos leves, el examen pulmonar en reposo suele ser normal y el único síntoma predominante puede ser la fiebre 4 . Sin embargo, hasta $27 \%$ de pacientes con infección por $\mathrm{VIH}$ que desarrollan neumonía por $P$. jiroveci pueden presentar insuficiencia respiratoria que requiere ventilación mecánica ${ }^{11}$.

La infección por $P$. jiroveci también ha sido descrita desde 1960 como un patógeno oportunista en niños inmunocomprometidos con leucemia aguda o inmunodeficiencias congénitas con alteración de la función de linfocitos $\mathrm{T}$ y en pacientes adultos en tratamiento inmunosupresor o en quimioterapia antineoplásica ${ }^{11,12}$. A principios de la década de los $80 \mathrm{~s}$, la neumonía por $P$. jiroveci fue la primera infección oportunista asociada al VIH, siendo enfermedad definitoria en más de la mitad de los adultos en etapa SIDA de E. U. A. ${ }^{12,13}$. Se estima que hasta $80 \%$ de los pacientes con recuentos de linfocitos CD4 $<200$ céls $/ \mathrm{mm}^{3}$ podrían desarrollar la enfermedad ${ }^{11}$.

La incidencia de neumonía por $P$. jiroveci ha disminuido significativamente con el uso de profilaxis y TARV. En pacientes en etapa SIDA de Europa Occidental y E. U. 
A. se estima en menos de un caso por 100 personas con infección por $\mathrm{VIH} / \mathrm{año}^{4}$. La mayoría de los casos ocurren en pacientes que desconocen su infección por VIH o que no están recibiendo profilaxis o TARV y aquellos que presentan inmunosupresión grave $\left(\mathrm{CD} 4<200 \text { céls } / \mathrm{mm}^{3}\right)^{4,12}$. Sin embargo, la incidencia estimada de neumocistosis extrapulmonar ha sido difícil de determinar y varía entre cifras menores de $0,5 \%$ a $2,5 \%$, este último con datos de autopsias entre 1980 a $1988^{1}$. El consenso general es que es una enfermedad con incidencia menor a $1 \%$.

A pesar del gran número de casos de neumonía por $P$. jiroveci desde la pandemia del VIH, la infección extrapulmonar de $P$. jiroveci es de rara ocurrencia. Raviglione ${ }^{1}$ en el año 1990 hace una revisión de los primeros 50 casos de neumocistosis extrapulmonar reportados desde 1954, de los cuales 34 casos estaban asociados a infección por VIH, 33 de ellos documentados entre 1987 a 1990. Todos ellos con inmunocompromiso grave $\mathrm{e}^{1,13}$.

La mayoría de los casos descritos son durante la década de los 90s, en los cuales no era claro si los pacientes VIH en etapa SIDA eran más susceptibles a una diseminación extrapulmonar que otros pacientes con riesgo de neumonía por P. jiroveci $i^{13,14}$. Sin embargo, tres factores podrían tener importancia, el primero es que los pacientes con infección por VIH logran sobrevivir más tiempo y pueden llegar a etapas de mayor inmunocompromiso. El segundo factor es la presencia de una disgammaglobulinemia lo que podría favorecer la migración de agentes desde el pulmón a otros sitios, apoyado en que la inmunidad humoral es crítica en prevenir la diseminación, esto relacionado con una disminución de fagocitosis y alteraciones en la opsonización, sugerido en estudios in vitro ${ }^{1,15}$. El tercer factor es el uso de pentamidina en aerosol como profilaxis primaria y secundaria de neumonía por $P$. jiroveci, ampliamente usada a finales de los 80 s y principios de los 90 s, mismo período en que se reportaron los primeros casos de neumocistosis extrapulmonar ${ }^{1}$. Se ha planteado que el uso de pentamidina en aerosol se distribuye sólo localmente en el pulmón y no protegería frente a una diseminación sistémica de $P$. jiroveci ${ }^{16}$. Sin embargo, y probablemente debido a lo poco frecuente de esta patología, no se ha logrado estimar un factor predictor de cuáles pacientes con neumonía por $P$. jiroveci tienen más riesgo de diseminación extrapulmonar, pero la mayoría de los pacientes reportados presentan CD4 $<50$ céls $/ \mathrm{mm}^{3(1,6)}$, a diferencia de individuos sin $\mathrm{VIH}$, donde el compromiso extrapulmonar es excepcional y sólo se ha encontrado en estudios post mortem ${ }^{6}$.

La neumocistosis extrapulmonar ha sido observada en varios órganos y tejidos ${ }^{1,6,13,15-19}$. La presentación es más frecuente en múltiples sitios en pacientes con neumonía por $P$. jiroveci concurrente. Puede corresponder a una enfermedad restringida a un sitio único o múltiples sitios no contiguos. Se ha descrito compromiso de pabellón auricular, coroides, retina, tiroides, paratiroides, hígado, bazo, páncreas, riñón, uréteres, glándulas adrenales, corazón, tráquea, ganglios linfáticos, pleura, líquido ascítico, orina, médula ósea, músculos, hipófisis, meninges, corteza cerebral, tracto gastrointestinal, incluyendo un caso de perforación de intestino delgado ${ }^{17}$. El sitio más frecuente de compromiso extrapulmonar corresponde a los ganglios linfáticos ${ }^{6-16}$. La vía de diseminación puede ser hematógena, linfática, y también por contigüidad como en casos descritos de neumonía por $P$. jiroveci con derrame pleural concomitante ${ }^{6-16}$.

El diagnóstico de neumocistosis extrapulmonar no es difícil una vez que es considerado como diagnóstico diferencial, sin embargo, rara vez es sospechado por su baja frecuencia, presentación clínica no específica que puede ser atribuido a una amplia variedad de otras infecciones o enfermedades oportunistas que ocurren en pacientes con VIH e inmunocompromiso grave $\mathrm{e}^{6,20,21}$. Una vez sospechada la diseminación extrapulmonar y ante la ausencia de un método de cultivo in vitro para $P$. jiroveci, es necesario demostrar sus quistes o trofozoitos en el o los tejidos afectados o en líquidos estériles ${ }^{6,22}$. Cabe mencionar que en muchos de los casos publicados se realizó el diagnóstico en forma incidental o post mortem, de los cuales varios pacientes presentaban otras infecciones o enfermedades en forma concomitante (CMV, Cryptococcus neoformans, Mycobacterium avium complex, Mycobacterium tuberculosis, candidiasis, sarcoma de Kaposi y hepatitis B) $)^{1,6,14,20}$. Por lo tanto, es difícil estimar la real implicancia de la infección extrapulmonar por $P$. jiroveci en relación a letalidad o pronóstico.

En cuanto al tratamiento, de la variedad de agentes antiPneumocystis no hay ninguno claramente asociado a un mejor o peor resultado ${ }^{6}$. Sin embargo, por su alta eficacia y amplia disponibilidad oral e intravenosa, cotrimoxazol constituye el fármaco de elección para el tratamiento y profilaxis de la infección por $P$. jiroveci ${ }^{22}$. Además con el uso de TARV se ha reducido significativamente la infección por Pneumocystis, incluso en ausencia de profilaxis específica ${ }^{23}$.

En el caso de nuestro paciente, el diagnóstico de neumocistosis extrapulmonar fue realizado de forma incidental mediante la demostración de quistes de $P$. jiroveci en colon, cuya muestra fue obtenida como parte del estudio de diarrea crónica en un paciente inmunocomprometido. El diagnóstico de neumonía por $P$. jiroveci se realizó por sospecha imagenológica y posterior confirmación mediante citología del LBA, sin síntomas respiratorios evidentes al momento del ingreso, ni signos de falla respiratoria durante su evolución, pese a los hallazgos descritos en las imágenes. Esto hace plantear una neumonía por $P$. jiroveci sin criterios de gravedad, donde el síntoma principal fue la fiebre, sin embargo, presentó una diseminación extrapulmonar. 
Esto último podría relacionarse más con la severidad del inmunocompromiso que con la gravedad de la neumonía por $P$. jiroveci, considerando el recuento de linfocitos CD4 $<50$ céls $/ \mathrm{mm}^{3}$ del paciente.

Respecto al LDH sérico, diferentes estudios han demostrado valores elevados de LDH en sueros de pacientes con neumonía por $P$. jiroveci, con una sensibilidad entre $82-100 \%{ }^{11}$, aunque la especificidad es baja ${ }^{4}$. No obstante, su interpretación debe ser prudente porque hay casos en que puede ser normal, como el de este paciente. Otros estudios han intentado relacionar los valores de LDH con sobrevida en pacientes con neumonía por $P$. jiroveci. En este sentido tendrían peor pronóstico aquellos con valores elevados de LDH al ingreso, o que aumente durante el tratamiento ${ }^{11,24}$

Lamentablemente no fue posible realizar un estudio histopatológico de las lesiones hepáticas descritas $(<10$ $\mathrm{mm})$ que podrían estar en el contexto de una hepatitis granulomatosa, descrita en casos de pacientes con diseminación hematógena de $P$. jiroveci ${ }^{6,25}$. El paciente inició en forma precoz TARV y cotrimoxazol en dosis terapéuticas para neumonía por $P$. jiroveci. Simultáneamente durante el estudio etiológico de la diarrea crónica presentó una franca y rápida mejoría a los pocos días de iniciado el tratamiento con cotrimoxazol, completando 21 días según lo recomendado para los casos de neumocistosis extrapulmonar, con buena respuesta clínica y disminución de parámetros inflamatorios.

\section{Resumen}

Comunicamos el caso de un varón de edad mediana, con diagnóstico reciente de infección por VIH en etapa SIDA, sin otras co-morbilidades, y cuadro subagudo de compromiso del estado general, fiebre, tos poco productiva autolimitada y diarrea crónica no sanguinolenta. Se realizó el diagnóstico de neumonía por Pneumocystis jiroveci mediante sospecha imagenológica y tinción de quistes de este patógeno en muestras de lavado broncoalveolar. Se inició tratamiento con cotrimoxazol y TARV con buena evolución clínica. En forma concomitante se realizó el estudio etiológico de diarrea crónica y a través del estudio histopatológico de mucosa colónica se observaron numerosas estructuras quísticas extracelulares, características de Pneumocystis por lo que se realizó el diagnóstico de neumocistosis extrapulmonar. La neumocistosis extrapulmonar es una causa infrecuente de infección por $P$. jiroveci, que requiere un alto índice de sospecha en pacientes con VIH e inmunocompromiso grave, en los cuales es frecuente la co-infección de infecciones oportunistas. Es perentorio realizar un diagnóstico etiológico y tratamiento precoz.

\section{Referencias bibliográficas}

1.- Raviglione M C. Extrapulmonary pneumocystosis: the first 50 cases. Rev Infect Dis 1990; 12: 1127-38.

2.- Thomas C F Jr, Limper A H. Pneumocystis pneumonia. N Eng J Med 2004; 350: 2487-98.

3.- Russian D A, Levine S J. Pneumocystis carinii pneumonia in patients without HIV infection. Am J Med Sci 2001; 321: 56-65.

4.- Panel on opportunistic infections in HIV-infected adults and adolescents. Guidelines for the prevention and treatment of opportunistic infections in HIV-infected adults and adolescents: recommendations from the Centers for Disease Control and Prevention, the National Institutes of Health, and the HIV Medicine Association of the Infectious Diseases Society of America. Disponible en: http://aidsinfo.nih.gov/ contentfiles/lvguidelines/adult_oi.pdf. (Fecha de acceso: 26 de enero de 2014).

5.- Morris A, Lundgren J D, Masur H, Walzer P D, Hanson D L, Frederick T, et al. Current epidemiology of Pneumocystis pneumonia. Emerg Infect Dis 2004; 10: 1713-20.

6.- Ng V L, Yajko D M, Hadley W K.
Extrapulmonary pneumocystosis. Clin Microbiol Rev 1997; 10: 401-18.

7.- Kovacs J A, Masur H. Evolving health effects of Pneumocystis: one hundred years of progress in diagnosis and treatment. JAMA 2009; 301: 2578-85.

8.- Medrano F J, Montes-Cano M, Conde M, de la Horra C, Respaldiza N, Gasch A, et al. Pneumocystis jirovecii in general population. Emerg Infect Dis 2005; 11: 245-50.

9.- Cerón I, Rabagliati R, Langhaus J, Silva F, Guzmán A M, Lagos M. Características clínicas, diagnósticas y pronósticas de pacientes con neumonía por Pneumocystis jiroveci en individuos infectados por el virus de la inmunodeficiencia humana e individuos inmunocomprometidos por otra etiología. Rev Chilena Infectol 2014; 31: 417-24.

10.- Huang L, Morris A, Limper A H, Beck J M. ATS Pneumocystis Workshop Participants. An oficial ATS Workshop Summary: Recent advances and future directions in Pneumocystis pneumonia. Proc Am Thorac Soc 2006; 3: 655-64.

11.- Calderón Sandubete E, de Armas Rodríguez Y, Capó de Paz V. Pneumocystis jirovecii: cien años de historia. Rev Cubana Med Trop 2011; 63: 97-116.
12.- Catherinot E, Lanternier F, Bougnoux M E, Lecuit M, Couderc L J, Lortholary O. Pneumocystis jirovecii pneumonia. Infect Dis Clin North Am 2010; 24: 107-38.

13.- Telzak E E, Cote R J, Gold J W, Campbell S W, Armstrong D. Extrapulmonary Pneumocystis carinii infections. Rev Infect Dis 1990; 12: 38086.

14.- Coker R J, Clark D, Claydon E L, Gompels M, Aisworth J G, Lucas S B, et al. Disseminated Pneumocystis carinii infection in AIDS. J Clin Pathol 1991; 44: 820-3.

15.- Masur H, Jones T C. The interaction in vitro of Pneumocystis carinii with macrophages and L-cells. J Exp Med 1978; 147: 157-70.

16.- Sundar K, Rosado-Santos H, Reimer L, Murray $\mathrm{K}$, Michael J. Unusual presentation of thoracic Pneumocysticcarinii infection in a patient with acquired immunodeficiency syndrome. Clin Infect Dis 2001; 32: 498-501.

17.- Kinchen K, Kinchen $T H$, Inglesby $T J r$. Pneumocystis carinii infection of the small intestine. J Natl Med Assoc 1998; 90: 625-27.

18.- Bartlett J A, Hulette C. Central nervous system pneumocystosis in a patient with AIDS. Clin Infect Dis 1997; 25: 82-5.

19.- Bellomo A R, Perlman D C, Kaminsky D L, Brettholz E M, Sarlin J G. Pneumocystis colitis 
in a patient with the acquired inmunodeficiency syndrome. Am J Gastroenterol 1992; 87: 75961.

20.- Northfelt D W, Clement M J, Safrin S. Extrapulmonary pneumocystosis: clinical features in human immunodeficiency virus infection. Medicine (Baltimore) 1990; 69: 39298.

21.- Fishman J A, Mattia A R. Case records of the Massachusetts General Hospital (Case 3-1995).
A 29-year-old man with AIDS and multiple splenic abscesses. N Engl J Med 1995; 332: 249-57.

22.- Calderón E J, Gutiérrez-Rivero S, Durand-Joly I, Dei-Cas E. Pneumocystis infection in humans: diagnosis and treatment. Expert Rev Anti Infect Ther 2010; 8: 683-701.

23.- Weverling G J, Mocroft A, Ledergerber B, Kirk O, González-Lahoz J, d'Arminio-Monforte A, et al. Discontinuation of Pneumocystis carinii pneumonia prophylaxis after start of highly active antiretroviral therapy in HIV-1 infection. Lancet 1999; 353: 1293-98.

24.- Boldt M J, Bai T R. Utility of lactate dehydrogenase vs radiographic severity in the differential diagnosis of Pneumocystis carinii pneumonia. Chest 1997; 111: 1187-92.

25.- Coash M, Farouhar F, Wu C H, Wu G Y. Granulomatous liver disease: a review. J Formos Med Assoc 2012; 111: 3-13. 